EPJ Web of Conferences 114. 02007 (2016)

DOI: 10.1051 /epjconf/20161140200 7

(C) Owned by the authors, published by EDP Sciences, 2016

\title{
PIV and LIF study of slot continuous jet at low Reynolds number
}

\author{
Zuzana Broučková ${ }^{1, a}$, Shu-Shen $\mathrm{Hsu}^{2}$, An-Bang Wang ${ }^{2}$ and Zdeněk Trávníček ${ }^{1}$ \\ ${ }^{1}$ Institute of Thermomechanics, the Czech Academy of Sciences, 18200 Prague, Czech Republic \\ ${ }^{2}$ Institute of Applied Mechanics, National Taiwan University, Taipei, Taiwan R.O.C.
}

\begin{abstract}
This study deals with a continuous jet issuing from a small narrow slot with a width of $0.36 \mathrm{~mm}$. The experimental arrangement is based on the piezoelectric synthetic jet actuator studied previously for easy comparisons. The working fluid is water at room temperature. The experiments were performed using methods of particle image velocimetry (PIV) and flow visualization (laser induced fluorescence, LIF). The time-mean volume flux through the exit nozzle was quantified using precise scales. The mean velocity and the Reynolds number were evaluated as $U_{\mathrm{m}}=0.12 \mathrm{~m} / \mathrm{s}$ and $R e=90$, respectively. The results of LIF and PIV techniques revealed the three-dimensional character of the flow field, namely the saddle-shape velocity profiles. This behavior is typical for steady jets from a rectangular nozzle. The obtained results were compared with previous measurements of the synthetic jet issuing from the same cavity and the slot nozzle.
\end{abstract}

\section{Introduction}

The turbulent continuous jets (CJ) issuing from planar and rectangular slots have been widely studied. The studies were focused on general characteristics of CJs (e.g. Gutmark and Wygnanski [1], Everitt and Robins [2], Tsuchiya et al. [3]), spatial developement of the velocity profile and the pressure distribution of the jet (Abramovich [4]), coherent structures in plane jets (e.g. Thomas and Goldschmidt [5]), axis-switching of the jet (Zaman [6]), saddle-back velocity profiles (Vouros et al. [7]), behavior of the jets at low Reynolds numbers (Sato and Sakao [8]) or the influence of an aspect ratio of the nozzle (Deo et al. [9]).

The synthetic jet (SJ) is a fluid flow generated from fluid oscillations during a periodical fluid exchange between an actuator cavity and surroundings. A meaningful advantage of SJs is a relative simplicity of an arrangement: neither blower nor fluid supply piping is required. SJs are investigated by means of experimental, theoretical and numerical methods - e.g. Smith and Glezer [10], Cater and Soria [11], Mallinson and Reizes [12].

This experimental study is focused on a continuous jet issuing at a low Reynolds number from a rectangular slot. The experimental setup is based on a previously studied piezoelectric SJ actuator (see [13], [14]) which was adapted to be able to work also in a continuous (steady) regime as is described thereinafter. The aim of this study is to verify the possibility of the device to work in continuous regime and also to obtain results which can be used as a benchmark for further experiments with SJs.
For this reason the operating parameters of the CJ were chosen to be similar to those of SJ.

\section{Parameters}

The continuous rectangular jet is described by the dimensions of the exit slot (the width $B$ and the length $L_{\mathrm{s}}$ ) and by the Reynolds number:

$$
R e=\frac{2 B U_{\mathrm{m}}}{v},
$$

where $U_{\mathrm{m}}$ is the mean exit velocity and $v$ is the kinematic viscosity.

The Reynolds number of SJs was defined [14] for the same rectangular slot $\left(B \times L_{\mathrm{s}}\right)$ as:

$$
R e_{S J}=\frac{2 B U_{0}}{v},
$$

where $U_{0}$ is the time-mean exit velocity:

$$
U_{0}=\frac{1}{T} \int_{0}^{T_{E}} u_{0}(t) d t,
$$

where $T=1 / f$ is the time period, $f$ is frequency, $T_{\mathrm{E}}$ is the extrusion time and $u_{0}(t)$ is the centerline exit velocity (see e.g. Smith, Glezer [10]).

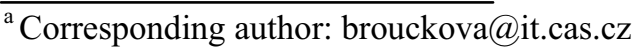




\section{Experimental investigation}

\subsection{Experimental setup}

Figure 1 shows a scheme of the experimental device. The experimental arrangement is based on the piezoelectric synthetic jet actuator. The actuator body is made out of PMMA (polymethyl methacrylate). It is equipped with a piezoelectric diaphragm (1), which is the piezoceramic transducer KPS-100 with the diaphragm diameter of $D_{\mathrm{D}}=$ $39.5 \mathrm{~mm}$. The cavity (2) diameter and depth are $44 \mathrm{~mm}$ and $6 \mathrm{~mm}$, respectively. The jet issues from a slot (3) on the top of the actuator. The slot width and length are $B=0.36 \mathrm{~mm}$ and $L_{\mathrm{s}}=40 \mathrm{~mm}$, respectively, i.e. the aspect ratio of the slot is $\mathrm{AR}=L_{\mathrm{s}} / B=111$. The length of the nozzle exit channel is $L=1.0 \mathrm{~mm}$. The actuator coordinate system $x, y, z$ is shown in figure 1 as well. The working fluid is water.

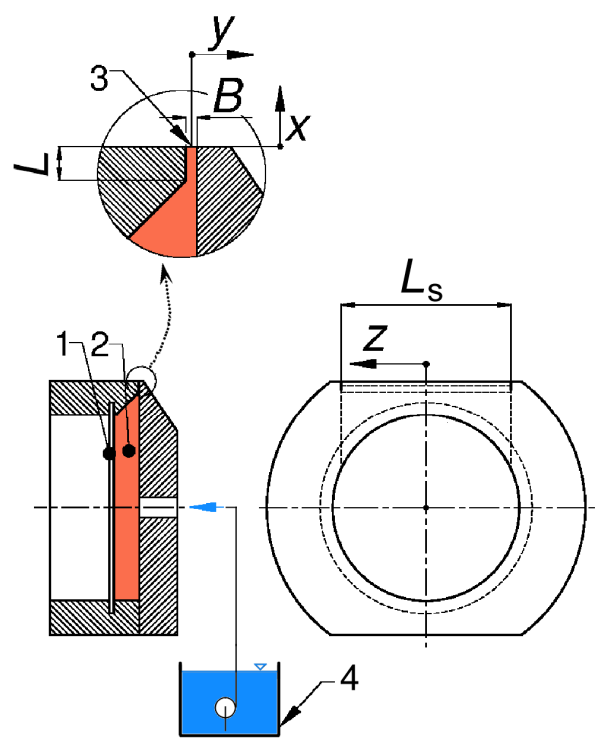

Figure 1. Investigated SJ actuator, 1: diaphragms, 2: cavity, 3: slot orifice, 4: pump.

The actuator can operate in two regimes: it can produce either the $\mathrm{CJ}$ or the SJ. When the device is operated in CJ regime, the fluid is fed into the actuator using centrifugal pump (4). The pump is connected with the actuator via the flexible pipe and the fluid enters the cavity opposite the center of the piezoelectric diaphragm - as is schematically shown in figure 1 . The pump is placed either in the same tank as the actuator or in the separate tank. The former arrangement is used for PIV experiments, the latter for LIF visualization as well as for evaluation of the time-mean volume flux by means of precise scales (as will be described further). In the case of $\mathrm{CJ}$ regime, the piezoelectric diaphragm is switched off.

When the device operates as a SJ actuator, the inlet from the pump is closed and the piezoelectric diaphragm is driven by the sinusoidal current with a drive frequency $f=46 \mathrm{~Hz}$ and RMS voltage $18.0 \mathrm{~V}$. A choice of the low frequency actuation at the near resonance frequency was refined recently by means of theoretical and experimental approaches - the details can be found in [13], [14]. Moreover, the time-mean exit velocity and the Reynolds number were evaluated in previous experiments [13], [14] as $U_{0}=0.17 \mathrm{~m} / \mathrm{s}$ and $R e=127$, respectively.

\subsection{Experimental methods}

Two experimental methods were used: laser induced fluorescence (LIF) visualization and particle image velocimetry (PIV). The former experiments were made in the Institute of Thermomechanics (Prague, Czech Republic) and the latter one in the Institute of Applied Mechanics (Taipei, Taiwan R.O.C.).

\subsubsection{LIF technique}

The experiments were made in a glass water tank with the dimensions: height $\times$ length $\times$ depth $=150 \times 245 \times 140$ $\mathrm{mm}$.

The LIF technique is based on the fluorescence effect. When a fluorescence dye is under a light with suitable wavelength, the dye absorbs the energy and consequently reemits part of it at higher wavelengths (see e.g. Seuntiens et al. [15] for more details). The Rhodamine B was chosen as a fluorescence dye (absorption wavelength from 460 to $590 \mathrm{~nm}$, emission wavelength from 560 to $670 \mathrm{~nm})$ - see [16]. Rhodamine B is usually used for temperature measurements because it has a temperaturedependent emission intensity. However, in the present study this effect was not used, the present experiments were approximately isothermal.

The experimental device was placed into the glass tank with pure, demineralised water, prepared in fourstage reverse osmosis water purification system. The pump was placed in a separate smaller tank. The separate tank was filled with dye solution (highly concentrated solution of Rhodamine B). Subsequently, the pump was turned on. The dye-stained flow was lit by laser sheet (Nd:Yag pulsed laser, Litron, NANO S 65-15, with a wavelength of $532 \mathrm{~nm}$ and an output of $65 \mathrm{~mJ}$, equipped with cylindrical optics). The laser sheet was perpendicular to the slot, i.e. in the plane of $x-y$, see figure 1 . The thickness of the laser sheet was about $1 \mathrm{~mm}$ in the test section.

The pictures of the visible flow field pattern (streaklines) were taken using digital camera (HiSense Neo, $2560 \times 2160$ pixels, 16 bit). The camera was equipped with lens (Tokina $100 \mathrm{~mm}$ F2.8 Macro D). To remove the laser light from the pictures, the colour filter (cut-off wavelength $570 \mathrm{~nm}$ ) was used. The camera was used to trigger the laser pulse. The measurement system was controlled using DynamicStudio v3.40 Software (Dantec Dynamics).

To evaluate the time-mean volume flux of the CJ, an auxiliary experiment was made using the precise scales (Mettler Toledo PR 8002 Delta Range). The actuator was placed into the separate tank and the amount of water discharged during the specified time was weighed.

The temperature of water was measured by a NTC thermistor sensor (Ahlborn AMR, Therm 2280-3). All experiments were made after thorough temperature equalization at room temperature. In this study, the temperature was $22^{\circ} \mathrm{C}$. 


\subsubsection{PIV technique}

The experiments were made in a cubic glass water tank with the side length of $390 \mathrm{~mm}$. During PIV measurement the pump was placed in the same tank as the actuator. Polyamide seeding particles (Dantec, $5 \mu \mathrm{m}$ in diameter) were used. The light source was a Nd:YAG dual laser head system (NewWave, $120 \mathrm{~mJ}$ at $532 \mathrm{~nm}$ ) with a cylindrical lens. The image pairs were acquired using a high resolution CCD camera (Kodak, MegaPlus ES1.0) with a spatial resolution of $1008 \times 1016$ pixels. The camera was equipped with the lens (Micro-Nikkor $105 \mathrm{~mm}$ ). The measurement system was controlled using Dantec Dynamics PIV system (FlowMap1500). The velocity vectors were consequently calculated using DynamicStudio v3.40 Software. The PIV adaptive technique was used. The minimum interrogation area was $32 \times 32$ pixels, the maximum interrogation area was $64 \times 64$ pixels. The resulting velocity maps were averaged from a sequence of 100 images.

Previously, the velocity field of the SJ was captured at selected phases of the velocity cycle and the additional apparatus was used for this reason - the details can be found in [14]. The resulting pictures were also averaged from a sequence of 100 images in the same phase, [14].

\section{Results and discussion}

Firstly, the time-mean volume flux $Q$ and the velocity $U_{\mathrm{m}}$ of the CJ was evaluated by means of the precise scales. The results are: $Q=0.00167 \mathrm{l} / \mathrm{s}$ and $U_{\mathrm{m}}=0.12 \mathrm{~m} / \mathrm{s}$. The corresponding Reynolds number is $R e=90$. These values are in a good agreement with the parameters of the previously studied SJ $[13,14]$.

Figure 2 shows a typical visualization of the jet (a) in the middle of the actuator slot $(x-y$ plane, $z=0)$ and $(b)$ along the $z$-axis $(z-x$ plane, $y=0)$.

The visualization in the middle of the actuator slots (figure 2a) shows the evolution of the jet and its gradual spreading. The influence of the geometry of the exit part of the cavity and the nozzle is visible - since the exit part of the cavity is not symmetrical against the nozzle and the nozzle exit channel is relatively short, $L=1.0 \mathrm{~mm}$ (see figure 1), the issuing jet is slightly deflected from the vertical direction to the right hand side. From the figure $2 \mathrm{~b}$ it is obvious that the distribution of the jet along the $z$ axis is not fully uniform. The flow in the surroundings of the midpoint of the slot $(z=y=0)$ is weaker then the flow at the off-axis parts (visible as darker area in the middle of the flow). This behavior is typical for 2D turbulent jets, the initially uniform jet creates two off axis peaks in proximity of the edges of the long size of the slot (here near the edges of the nozzle at $z=+L_{\mathrm{s}} / 2$ and $z=-$ $L_{\mathrm{s}} / 2$ ), see e.g. Vouros et al. [7]. In the present experiment the behavior is probably also supported by the confined 3D geometry of the cavity (namely, the cylindrical cavity with an axial inlet and a radial outlet) and by the short nozzle exit channel.

Streamwise velocity maps at the distances $x /(2 B)=5$, 10,15 and 20 are presented in figure 3 . The maps were evaluated from the velocity profiles, which were measured at the planes of $z=(-15,-10,-5,0,5,10,15)$ $\mathrm{mm}$. At the coordinates $z=-25 \mathrm{~mm}$ and $z=25 \mathrm{~mm}$ the zero values were artificially added in the pictures. The measurement confirms the expected character of the jet two off axis velocity maxima are visible at the distances $z$ $=-15 \mathrm{~mm}$ and $z=15 \mathrm{~mm}$ while the gradual decrease of the velocity in the center part of the jet is apparent. The resulting velocity profiles are known as saddle-back profiles of CJs (see e.g. Vouros et al. [7]). Note that this effect was identified for SJs too - see Smith and Glezer [10].
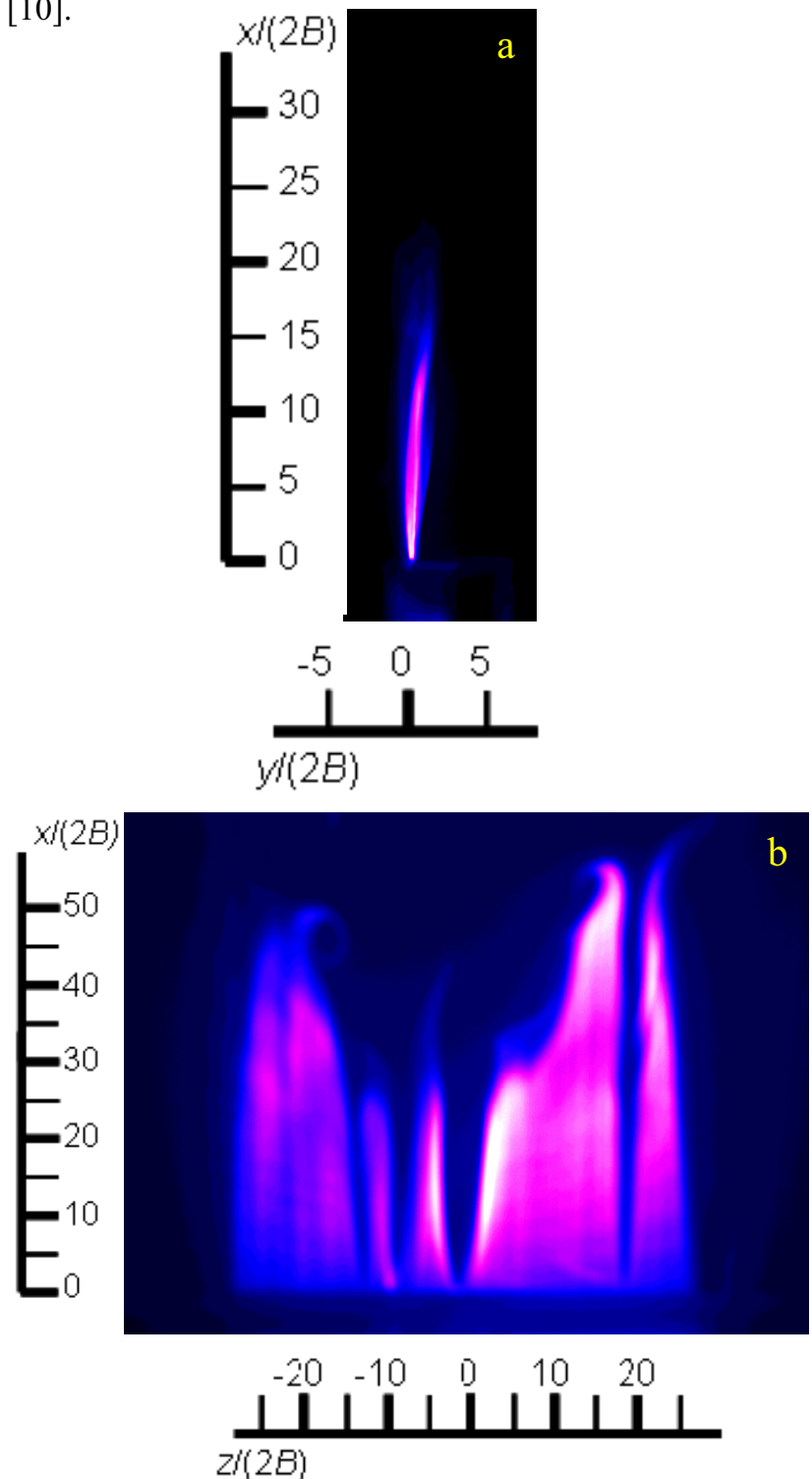

Figure 2. LIF visualization of the CJ

(a) In the middle of the actuator slot $(z=0)$, (b) Along the $z$-axis.

It is worth noting here that the measurement using PIV in a close vicinity of a body surface is influenced by the unavoidable reflection of the laser beam from the surface (the near wall effect). Moreover, the diameter of the chosen particles is relatively large for the present geometry - namely, the particle diameter is only two orders lower than the lateral dimension of the slot $(B)$, i.e. the initial lateral dimension of the jet. This fact can influence the ability of the particles to follow the flow properly. Moreover, the slot width $B$ is approximately 
three times narrower that the laser sheet thickness. For these reasons the PIV measurement undervalues the velocity magnitude in the near field - approximately for the distance $x /(2 B)<6$. It is obvious from figure 3 , the maximum velocity at the distance $x /(2 B)=5$ is unrealistically low - even lower than maximum velocities for $x /(2 B)=10$.

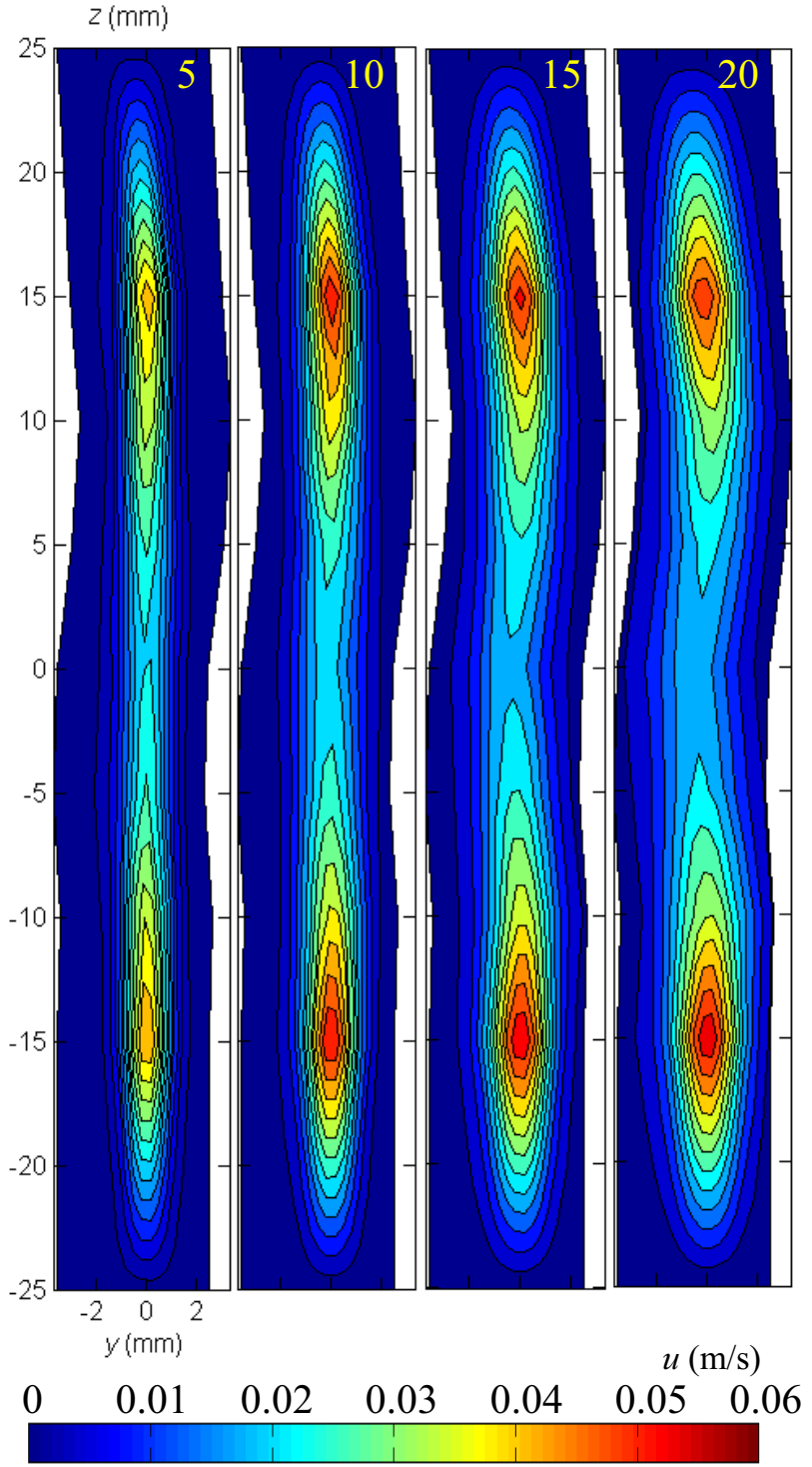

Figure 3. Streamwise velocity maps of the CJ (measured at distances $x /(2 B)=5,10,15,20)$.

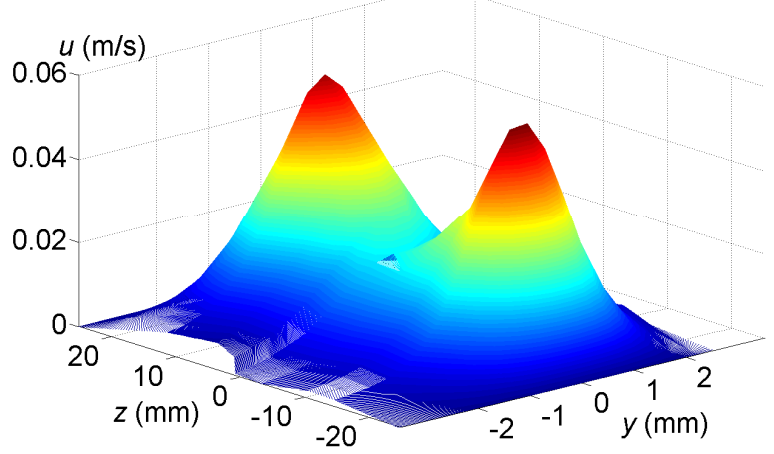

Figure 4. Streamwise velocity map at $x /(2 B)=15$.
For an illustrative purposes the streamwise velocity map at $x /(2 B)=15$ is also shown as three-dimensional contour plot in figure 4 . The character of the jet is clearly visible - two off axis peaks near the ends of the nozzle slot $\left(z=+L_{\mathrm{s}} / 2\right.$ and $\left.z=-L_{\mathrm{s}} / 2\right)$ and the lower velocity in the center of the jet create a typical saddle-shaped profile.

Figure 5 shows the velocity profiles (namely, the streamwise component of the velocity) in the dimensionless form. The horizontal coordinate is normalized by the half-width of the jet $y_{0.5}$ (it will be discussed in the following paragraph). The theoretical profile according to Blevins [17] is also shown, $u / u_{\max }=$ $\left.\exp \left[\begin{array}{lll}-57 & (0.11 & y / y_{0.5}\end{array}\right)^{2}\right]$. The measured profiles collapse on one curve which agrees excellently with the theoretical one. The deviations of little import around the distance $y / y_{0.5}=-2$ are caused by the above mentioned deflection of the jet to the right hand side.

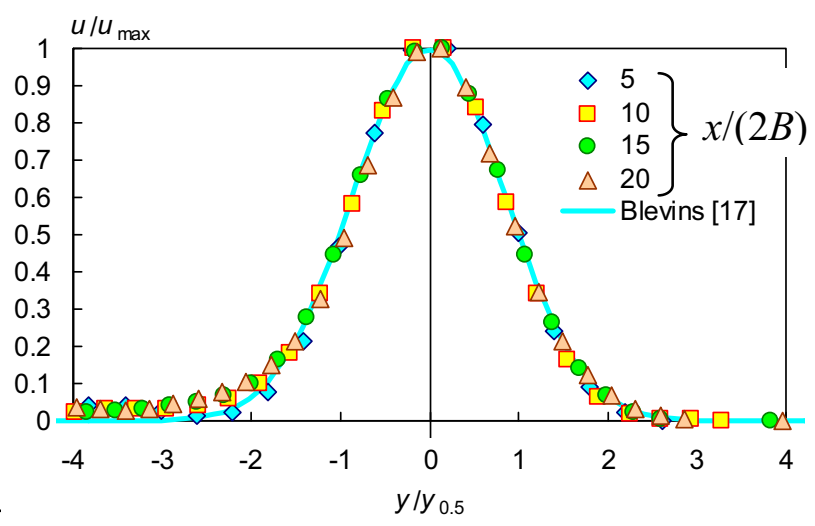

Figure 5. Streamwise velocity profiles.

Figure 6 presents the downstream evolution of the half-width of the jet. The curve for turbulent $2 \mathrm{D}$ jet according to Blevins [17] is plotted for comparison purposes, $y_{0.5} /(2 B)=0.11 x /(2 B)$. As expected the halfwidth increases gradually with the axial coordinate $x$ as the surrounding fluid is entrained into the jet. It can be seen that the half-width depends on the spanwise location - the half-width decrease gradually with the coordinate $z$ towards borders of the jet. The results for $x /(2 B) \geq 10$ reasonably agree with the curve by Blevins. However the inclination of the present results is smaller. The main reason can be a relatively low Reynolds number of the examined jet with a transition from laminar to turbulent flow. Moreover, a relatively small aspect ratio can cause another difference from behavior of plane jets [17]. Note that the relationship by Blevins [17] is considered for fully turbulent flow $(\operatorname{Re}>3000)$ and infinite length of the slot. The experimental data by Vouros et al. [7] $(R e=$ 46000, AR = 10) and Deo et al. [9] $(R e=3000, \mathrm{AR}=61)$ are also shown in figure 6 . The present data reasonably agree (for $x /(2 B) \geq 10$ ) with these results $[7,9]$, although the operation parameters of [7] and [9] were quite different.

Note that the PIV results for $x /(2 B)=5$ are influenced by the near wall effect, as was explained in the text above. The region contaminated by the near wall effect is marked as a grey area in figure 6 (and similarly in the following figures).

Volumetric flow rate at the nozzle exit is defined as 


$$
Q_{0}=\int_{-}^{L_{s}} L_{s} / 2 \int_{-B / 2}^{B / 2} u(y, z) d y d z=\int_{-}^{L_{s} / 2} L_{s}(z) d z .
$$

Figure 7 shows the volumetric flow rate relative to the $z$ coordinate, $Q_{z}$. It is obvious that the entrainment of the flow is highly dependent on the spanwise location. Mixing with the surroundings is very intensive near the borders of the jet ( $z=-15 \mathrm{~mm}$ and $z=15 \mathrm{~mm}$ ) because of fluid entrainment in both lateral and spanwise direction in the shear layers. Over against the entrainment around the centerline (i.e. around $z=0$ ) is minimal.

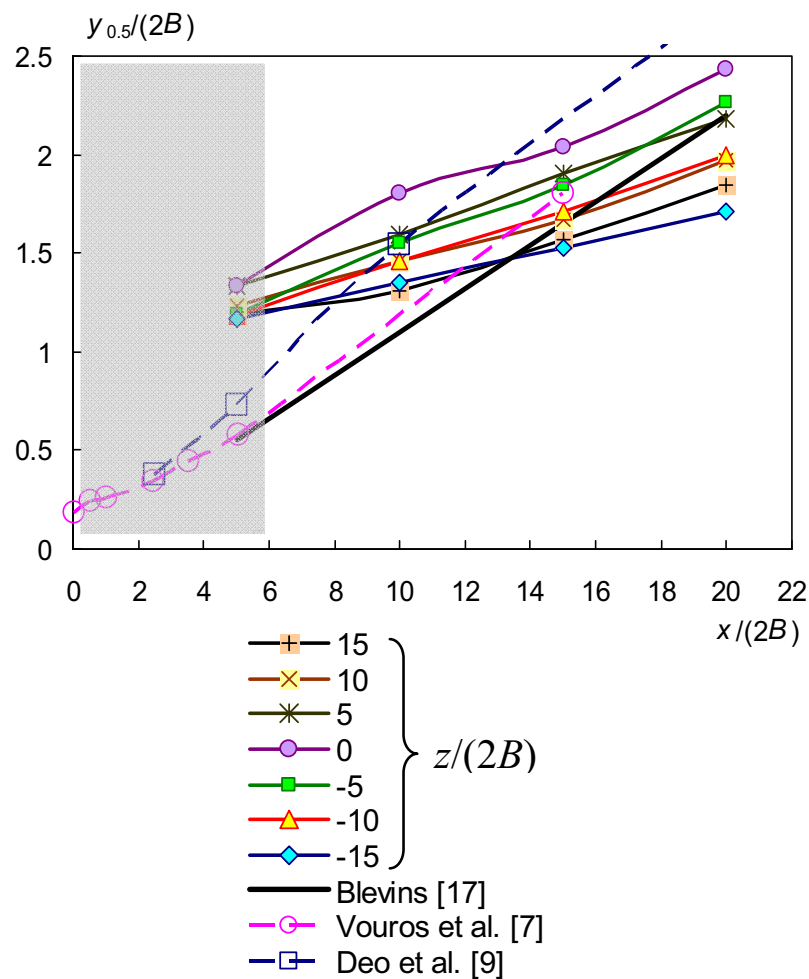

Figure 6. Half-width of the $\mathrm{CJ}$; grey area depicts the near wall effect in PIV experiments.

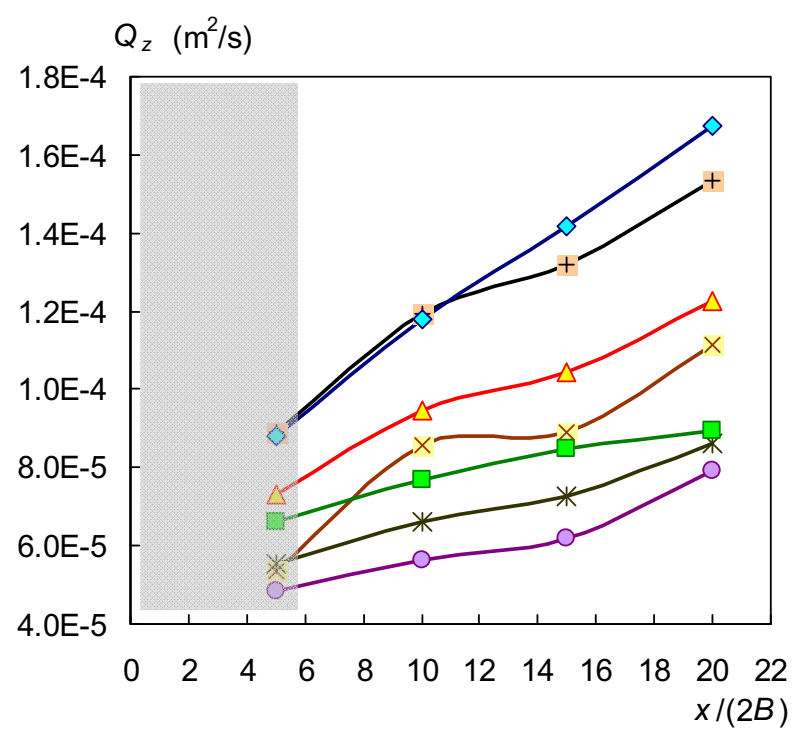

Figure 7. Volumetric flow rate relative to $z$ coordinate $Q_{z}$; grey area depicts the near wall effect in PIV experiments; for legend see figure 6 .
Figure 8 shows the comparison between the CJ and SJ velocity vector field $(z=-15 \mathrm{~mm})$. The $\mathrm{SJ}$ is shown in the blowing part of the cycle; more details were described previously $-[13,14]$. The pulsatile character of the SJ is visible, mainly in the near field $(x /(2 B)<10)$. The SJ has a higher velocity at the axis and the jet is spread more than the CJ. The distinct deflections of the jets to the right hand side can be observed. This effect is stronger for SJ than for CJ. The reasons are a non-symmetrical geometry of the nozzle, as was written in the text above. Note the above discussed near wall effect is manifested in figure 8 as an undervaluation of the velocity magnitude in the near field for $x /(2 B)<5$.

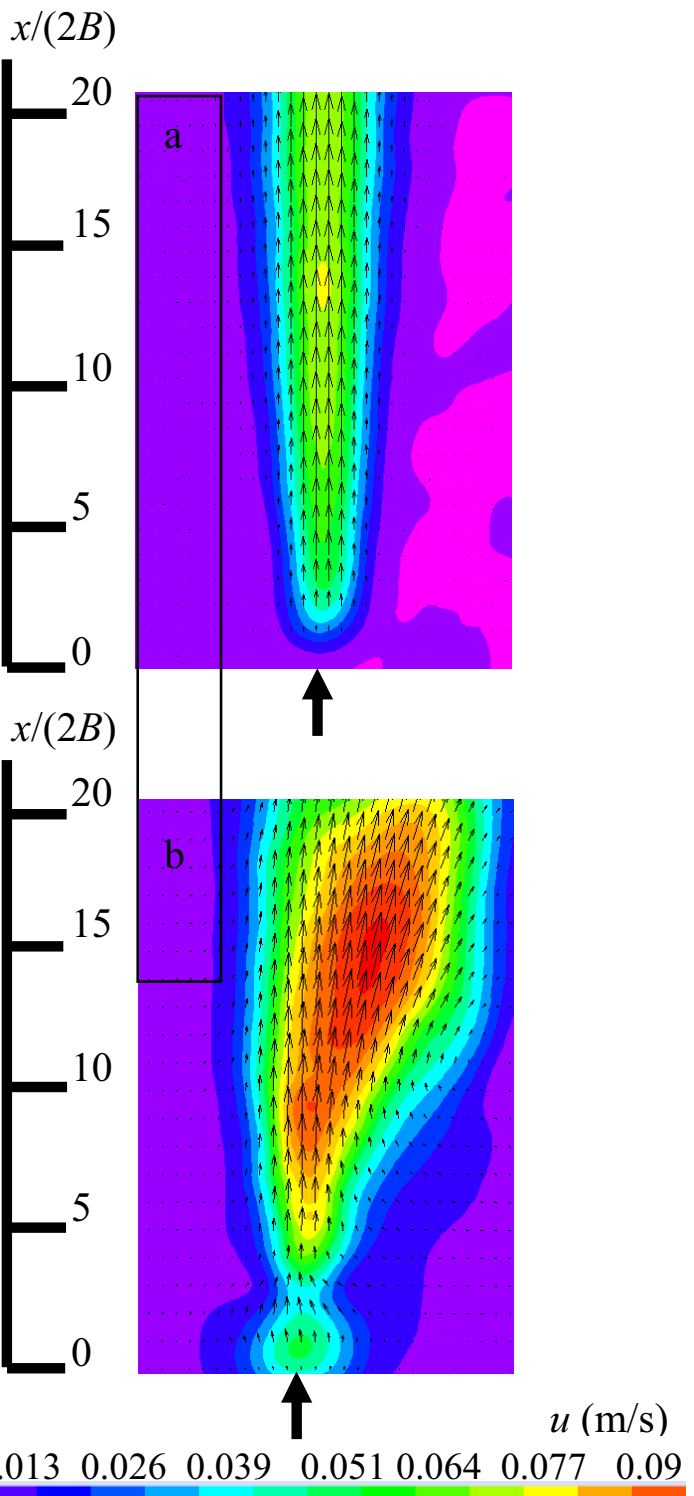

Figure 8. Velocity vector maps
(a) CJ
(b) $\mathrm{SJ}$
The arrow depicts the position of the nozzle axis, $y=0$.

Finally, figure 9 presents an evolution of the velocity magnitude along the centerline $(y=z=0)$. The velocity magnitude of the CJ and the time-mean velocity magnitude of the SJ are compared. The line for turbulent $2 \mathrm{D}$ jets according to Blevins [17] $\left(u \sim x^{-0.5}\right)$ is plotted too. 
The velocity of the $\mathrm{CJ}$ reaches its maximum approximately at $x /(2 B)=10$. The reason of this unexpected result can be the above discussed near wall effect - see a grey area in figure 9 .

More downstream, the velocity of CJ remains constant till $x /(2 B)=18$ and than starts to gradually decrease. On the other hand, the SJ velocity reaches the maximum approximately at the same distance $(x /(2 B)=$ $10)$ and than immediately drops. The SJ velocity decays at first with $x^{-0.5}$, further downstream the decay is faster $\left(x^{-0.78}\right)$ - see figure 9 .

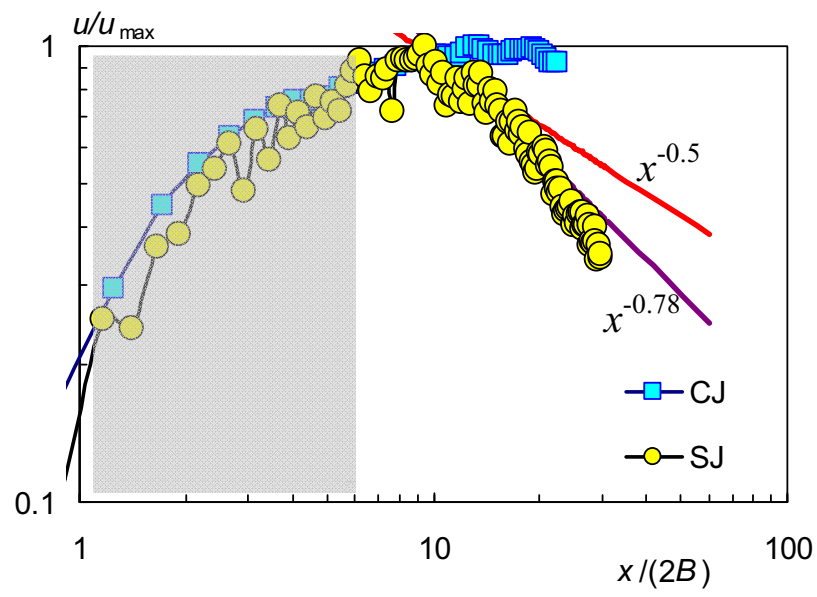

Figure 9. Centerline velocity decay; grey area depicts the near wall effect in PIV experiments.

\section{Conclusions}

The water continuous jet issuing from a small narrow slot $(0.36 \mathrm{~mm}$ width $)$ was experimentally studied. The experimental arrangement was based on the previously investigated piezoelectric synthetic jet actuator. The experiments using LIF and PIV techniques confirmed expected character of the jet: the three-dimensional character of the flow field was observed and the saddleback velocity profiles were found. The comparison with previously studied synthetic jet was performed. The jet flows with the saddle-back velocity profiles are known for the continuous jets as well as for the synthetic jets. This effect is promoted by the present geometry, mainly by the $3 \mathrm{D}$ geometry of the cavity (the cylindrical cavity with an axial inlet and a radial outlet) and by a relatively short nozzle exit channel.

\section{Acknowledgments}

We gratefully acknowledge the support of the Grant Agency of the Czech Republic - Czech Science Foundation (project no. P101/14-08888S) and by the Czech-Taiwanese Joint Research Project under the Agreement on scientific cooperation between the Academy of Sciences of the Czech Republic and the National Science Council - Ministry of Science and Technology in Taiwan (PPP project no. MOST/14/001).

\section{References}

1. E. Gutmark, I. Wygnanski, J. Fluid Mech. 73 (1976)

2. K.W. Everitt, A.G. Robins, J. Fluid Mech. 88 (1978)

3. Y. Tsuchiya, C. Horikoshi, T. Sato, Exp. Fluids 4 (1986)

4. G.N. Abramovich, Int. J. Heat Mass Transfer 25, 12 (1982)

5. F.O. Thomas, V.W. Goldschmidt, J. Fluid Mech. 163 (1986)

6. K.B.M.Q. Zaman, J. Fluid Mechanics 316 (1996)

7. A. P. Vouros, T. Panidis, A. Pollard, R. Schwab, Int. J. Heat Fluid Flow 51 (2015)

8. H. Sato, F. Sakao, J. Fluid Mech. 20 (1964)

9. R. C. Deo, J. Mi, G. J. Nathan, Phys. Fluids 20 (2008)

10. B.L. Smith, A. Glezer, Phys. Fluids 10 (1998)

11. J.E. Cater, J. Soria, J. Fluid Mech. 472 (2002)

12. S.G. Mallinson, J.A. Reizes, G. Hong, Aeronaut. J. 105, 1043 (2001)

13. Z. Broučková, T. Vít, Z. Trávníček, EPJ Web of Conferences 92, 02007 (2015)

14. Z. Broučková, S-S. Hsu, A-B. Wang, Z. Trávníček, Advanced Materiál Research 1104 (2015)

15. H.J. Seuntiëns, R. N. Kieft, C.C.M. Rindt, A.A. van Steenhoven, , Exp. Fluids 31, 5 (2001)

16. Planar-LIF software, Installation \& user's guide, Dantec Dynamics (2002)

17. R. D. Blevins, Applied Fluid Dynamics Handbook. (Florida: Krieger Publishing Company, 2003) 